\title{
Space-Time Cooperation Diversity Using High-Rate Codes
}

\author{
GENYUAN WANG, YIMIN ZHANG and MOENESS G. AMIN \\ Center for Advanced Communications, Villanova University, Villanova, PA 19085, USA \\ E-mails: genyuan.wang@villanova.edu,yimin.zhang@villanova.edu,moeness.amin@villanova.edu
}

\begin{abstract}
In a multi-user communication system, cooperative diversity allows single-antenna mobile sets to achieve transmit diversity. Cooperative diversity improves the communication capacity and enhances the robustness of a wireless link when a single channel alone is not reliable. In this paper, a novel cooperative diversity scheme is introduced that enables simultaneous transmission of non-redundant data from all cooperative terminals. By taking advantages of both high-rate full-diversity space-time codes and the cooperative diversity, the proposed method provides high diversity gain beyond the number of physical transmit antennas without compromising the data rates. In essence, the proposed system aims at higher data rates over the non-cooperative counterpart, while maintaining the full diversity gain.
\end{abstract}

Keywords: cooperative diversity, space-time coding, wireless networks, mobile communications, MIMO

\section{Introduction}

The multi-input-multi-output (MIMO) methodology, by using multiple transmit and receive antennas and incorporating appropriate space-time coding schemes, allows considerable increase of the information capacity that a transmitter can deliver to a receiver [1-4]. A MIMO system is particularly effective when the channels are high rank. This happens when the correlations among different channel coefficients are low. Without increasing premium bandwidth or transmit power, a MIMO system can achieve higher diversity gain and increase the data rates, compared to a single-input-single-output (SISO) system.

It is often impractical, however, for a transmitter or a receiver to have the required number of antennas to achieve a desirable diversity gain. In addition, direct delivery of information from a transmitter to a receiver may not be effective when they are far apart, or when the wireless communication links are highly impaired. This typically occours when considering a broad class of wireless networks, such as local area networks (LANs), ad hoc networks, and sensor networks. For such applications, cooperative diversity exploiting cooperation among multiple terminals becomes a promising technique.

Cooperative diversity techniques have attracted considerable and increasing attentions over the recent several years. In responding to the increasing needs of effective and reliable wireless networks in various application scenarios, the development of cooperative diversity techniques have benefitted from the recent advances of space-time coding, transmit diversity, and MIMO technologies. Despite the active efforts in cooperative diversity and networks, however, the research so far have focused primarily on the feasibility, protocols, and capacity analysis $[5,6,7,8,9,10,11]$. Proper design of distributed space-time codes, although being crucial to make the ideas practically amendable, has so far received little attention. High performance 
space-time cooperation schemes have focused on achieving the maximum capacity without properly addressing the implementation of the codewords.

In this paper, we develop an effective distributed space-time modulation scheme for cooperative wireless networks. In particular, we focus on the integrated approaches to design and optimize distributed space-time codes and cooperation protocols for effective data transmission, that is, achieving high bit rate per unit power, over wireless networks. We develop a novel protocol that permits the use of high-rate full-diversity codes in cooperative diversity systems and consider the optimization of the transmit power allocations. We mainly consider the amplify-and-forward algorithm, although the proposed scheme is also applicable to the decode-and-forward algorithm straightforwardly. In this paper, we focus on the situations where the channel state information (CSI) is known at the destination receiver. However, the proposed method is also applicable to differential cooperative diversity where the CSI is unavailable at the destination receiver [12].

This paper is organized as follows. Section 2 introduces the system model. The existing cooperative diversity algorithms and protocols are briefly reviewed in Section 3. In Section 4, after reviewing the high-rate full-diversity MIMO space-time code design, we propose a new space-time cooperation protocol that is applicable for the amplify-and-forward algorithm to effectively incorporate the high-rate full-diversity space-time codes. Section 5 examines the optimum power allocation over different portions of a distributed space-time code. Numerical results are given in Section 6.

\section{System Model}

To illustrate the concept of cooperative diversity in a wireless network, consider a simple model as depicted in Figure 1(a). A user cooperates with other users and serves as a relay terminal for them. Therefore, each user receives an attenuated and noisy version of the partner's transmitted signal and relays it to the destination or other relays. The destination receives a noisy version of the sum of the attenuated signals from all users. It is assumed that the terminals are half-duplex, i.e., a terminal does not receive signals when it transmits. It is also assumed that the fading channels remain constant over a period of several time slots, depending on the protocol and the number of cooperating users, and are observed over time to form independent stationary ergodic stochastic processes. It is assumed that the source and relay terminals are synchronized and the channels experience frequency non-selective (flat) fading.

The cooperation process can be divided into two phases (refer to Figure 1(b)). In the first phase (broadcast phase), the information is transmitted from a source user to the relay terminals, and the destination may also receive a copy of the same information. In the second phase (relay phase), the relay terminals transmit the signal to the destination. As we discuss below, the source user may or may not transmit information at the relay phase, depending on the protocols.

\section{Cooperative Diversity Algorithms and Protocols}

\subsection{Amplify-and-Forward and Decode-And-Forward Algorithms}

Depending on how a relay terminal relays the signals from other terminals, there are two major algorithms, namely, amplify-and-forward and decode-and-forward. In the amplify-andforward algorithm, a relay terminal amplifies the attenuated and noisy signals it receives and 

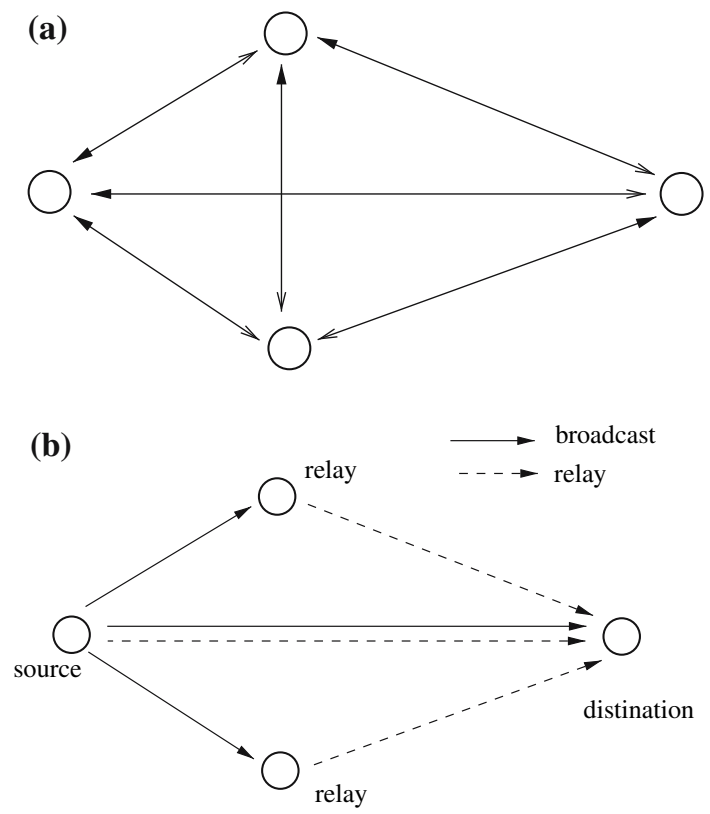

Figure 1. System model.

retransmits them to the destination as well as other possible relay terminals. The operation at the relay terminal is limited to amplification and, in some cases, some simple computations such as complex conjugation which is required in certain orthogonal codes including Alamouti's. On the other hand, when the decode-and-forward algorithm is used, the information is first decoded at a relay terminal, and then retransmitted from the delay terminal after proper coding. At the expense of introducing additional decoding operation at the relay terminals, the decode-and-forward algorithm allows the removal of relay noise (i.e., the noise that the relay terminals receive), and provides the flexibility of encoding the information at the relay phase so that higher spectral efficiency can be achieved $[8,13]$. In this paper, we focus on the amplify-and-forward algorithm.

\subsection{PROTOCOLS}

Several cooperative diversity protocols have been investigated. The early protocols use repetition-based approaches, which is illustrated in Figure 2(a). Assume that there are $M$ terminals (i.e., one source terminal and $M-1$ relay terminals). After the source broadcasts its information to the destination and the relays, all relays repeat this information in a sequential order. Therefore, a diversity gain of up to $M$ is achieved at the expense of reducing the degrees-of-freedom (DOFs) by a factor of $M$. Arbitrary permutations of these allocations in time and frequency does not alter the performance, as long as causality is preserved and each of the subchannels contains a fraction of $1 / M^{2}$ of the total DOFs in the channel. Such protocols does not require decoding and encoding at the relay terminals and, therefore, is suitable for the amplify-and-forward algorithm. The most significant disadvantage of such protocol is their low throughput rates. As is clear from Figure 2(a), transmission between different nodes at each time slot utilizes a fraction of $1 / M$ of the total DOFs in the channel. 

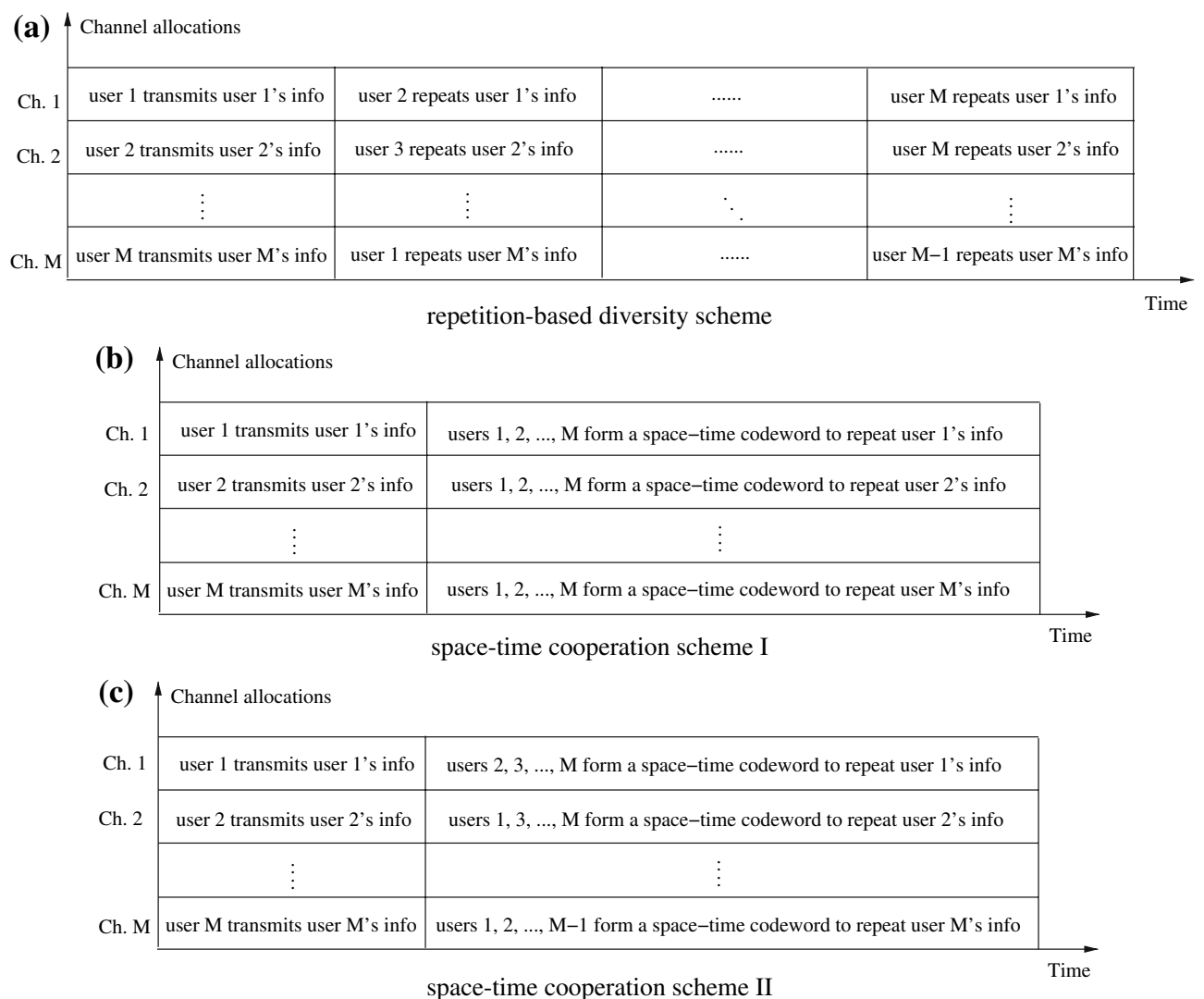

Figure 2. Cooperative diversity schemes.

Recently, more effective protocols have been developed to take advantages of the advances of MIMO space-time codes. For example, the space-time cooperation protocols proposed in $[8,13]$ are illustrated in Figure 2(b) and (c). These protocols provide higher spectral efficiency, particularly when the number of cooperative users is large. These protocols allow the use of a fraction of up to $1 / 2$ of the total DOFs in the channel. These protocols require decoding of other users' information and, therefore, should be incorporated with the decode-and-forward algorithm.

\section{High-Efficiency Space-Time Code Design}

In this section, we first review the general criteria of MIMO space-time code design and some high-rate full-diversity space-time codes. We then propose a new space-time cooperation protocol that incorporates the high-rate full-diversity space-time codes and is applicable to the amplify-and-forward algorithm for effective cooperative diversity transmission.

\subsection{Space-Time Codes in Mimo Systems}

Consider a transmitter that is equipped with $N_{t}$ transmit antennas, and the following $N_{t} \times N_{t}$ square codeword 


$$
\mathbf{C}=\left[\begin{array}{cccc}
c_{1}^{1} & c_{2}^{1} & \cdots & c_{N_{t}}^{1} \\
c_{1}^{2} & c_{2}^{2} & \cdots & c_{N_{t}}^{2} \\
\vdots & \vdots & \ddots & \vdots \\
c_{1}^{N_{t}} & c_{2}^{N_{t}} & \cdots & c_{N_{t}}^{N_{t}}
\end{array}\right]
$$

is transmitted. In a codeword, the rows of the coding matrix stand for "space" (i.e., antennas), and the columns stand for "time". When codeword $\mathbf{C}$ is transmitted, the probability that a maximum-likelihood receiver decides erroneously in favor of another signal codeword $\mathbf{E} \neq \mathbf{C}$ in independent Rayleigh fading channels is bounded by [2]

$$
P(\mathbf{C} \rightarrow \mathbf{E}) \leq\left(\prod_{i=1}^{r} \lambda_{i}\right)^{-N_{r}}\left(E_{S} / 4 N_{0}\right)^{-r N_{r}},
$$

where $r$ is the rank of $\mathbf{D}_{\mathbf{C E}}=\mathbf{C}-\mathbf{E}, \lambda_{i}, i=1, \ldots, r$, are the $r$ non-zero eigenvalues of $\mathbf{D}_{\mathbf{C E}} \mathbf{D}_{\mathbf{C E}}^{H}, N_{r}$ is the number of receive antennas, $E_{s}$ is the average transmitted symbol energy, and $N_{0} / 2$ is the noise variance in each of the two dimensions.

There are several criteria for MIMO space-time code design. The most commonly used ones are the rank criterion, determinant criterion, and symbol rate criterion [2]. In the next subsection, we consider high-rate, high-diversity code design that achieves large minimum determinant values.

\subsection{Full-Rate Full-Diversity Space-Time Code Design}

With these design criteria in mind, several space-time codes have been developed to achieve full rate and full diversity gain [14-21]. In this paper, a full-rate space-time code is defined as a code that carries $N_{t} \times L$ symbols of information over an $L$-symbol time period, yielding a symbol rate of $R=N_{t}$. In the following, we summarize the high-dimensional lattice-based systematic cyclotomic space-time codes proposed in [19, 20].

For a transmitter equipped with $N_{t}$ transmit antennas, the relationship $N_{t}=\phi(N) / \phi(n)$ holds, where $N=m n$, with $m$ and $n$ being two positive integers, and $\phi(n)$ denotes the Euler number of $n[19,20]$. For those combination of $m$ and $n$, there are $N_{t}$ distinct integers $n_{1}, n_{2}, \ldots, n_{N_{t}}, 0=n_{1}<\cdots<n_{N_{t}} \leq N-1$, such that $1+n_{i} m$ and $N$ are co-prime for $i=1,2, \ldots, N_{t}$. An $N_{t} \times N_{t}$ cyclotomic lattice generating matrix is defined as $[19,20]$

$$
\mathbf{G}(m, n)=\left[\begin{array}{cccc}
\zeta_{N} & \zeta_{N}^{2} & \cdots & \zeta_{N}^{N_{t}} \\
\zeta_{N}^{1+n_{2} m} & \zeta_{N}^{2\left(1+n_{2} m\right)} & \cdots & \zeta_{N}^{N_{t}\left(1+n_{2} m\right)} \\
\vdots & \vdots & \ddots & \vdots \\
\zeta_{N}^{\left(1+n_{N_{t}} m\right)} & \zeta_{N}^{2\left(1+n_{N_{t}} m\right)} & \cdots & \zeta_{N}^{N_{t}\left(1+n_{N_{t}} m\right)}
\end{array}\right]
$$

where $\zeta_{N}=\exp (j 2 \pi / N)$. A cyclotomic lattice $\Gamma_{N_{t}}(\mathbf{G}(m, n))$ is a set of $\left[x_{1}, x_{2}, \ldots, x_{N_{t}}\right]^{T} \subset$ $\mathcal{C}^{N_{t}}$, where superscript $T$ denotes transpose,

$$
\left[v_{1}, v_{2}, \ldots, v_{N_{t}}\right]^{T}=\mathbf{G}(m, n)\left[u_{1}, u_{2}, \ldots, u_{N_{t}}\right]^{T},
$$

and

$$
\left[\begin{array}{l}
\operatorname{Re}\left(u_{l}\right) \\
\operatorname{Im}\left(u_{l}\right)
\end{array}\right]=\left[\begin{array}{cc}
1 & 0 \\
\cos (2 \pi / m) & \sin (2 \pi / m)
\end{array}\right]\left[\begin{array}{l}
w_{l 1} \\
w_{l 2}
\end{array}\right],
$$


$l=1,2, \ldots, N_{t}$. In the above equation, $\operatorname{Re}(\cdot)$ and $\operatorname{Im}(\cdot)$ denote the real and imaginary part operators, respectively, and $w_{l 1}$ and $w_{l 2}$ are real integers which bear the source information.

Vector $\left[v_{1}, v_{2}, \ldots, v_{N_{t}}\right]^{T}$ obtained from (3) forms the following single-layer cyclotomic space-time code,

$$
\mathbf{V}=\left[\begin{array}{cccc}
v_{1} & 0 & \cdots & 0 \\
0 & v_{2} & \cdots & 0 \\
\vdots & \vdots & \ddots & \vdots \\
0 & 0 & \cdots & v_{N_{t}}
\end{array}\right]
$$

In a single-layer space-time code, the $N_{t} \times N_{t}$ codeword corresponds to $N_{t}$ complex source symbols $u_{1}, u_{2}, \ldots, u_{N_{t}}$, yielding a unit symbol rate $(R=1)$.

A cyclotomic space-time code can be arranged to form a multi-layer code which bears higher or even full rate information [14-20]. Denote $\left[v_{1}^{(k)}, v_{2}^{(k)}, \ldots, v_{N_{t}}^{(k)}\right]^{T}$ as the vector obtain from the $k$ th block of the aforementioned transform process, then a full $N_{t}$-layer space-time code can be expressed as

$$
\mathbf{V}=\left[\begin{array}{cccc}
\rho_{1} v_{1}^{(1)} & \rho_{2} v_{1}^{(2)} & \ldots & \rho_{N_{t}} v_{1}^{\left(N_{t}\right)} \\
\rho_{N_{t}} v_{2}^{\left(N_{t}\right)} & \rho_{1} v_{2}^{(1)} & \cdots & \rho_{N_{t}-1} v_{2}^{\left(N_{t}-1\right)} \\
\vdots & \vdots & \ddots & \vdots \\
\rho_{2} v_{N_{t}}^{(2)} & \rho_{3} v_{N_{t}}^{(3)} & \cdots & \rho_{1} v_{N_{t}}^{(1)}
\end{array}\right]
$$

where $\rho_{i}$ are some complex numbers (see $[20,21]$ for the determination of values of $\rho_{i}$ ). When all the $N_{t}$ layers are used to form a space-time code, the $N_{t} \times N_{t}$ codeword corresponds to $N_{t}^{2}$ source symbols, and the symbol rate becomes $R=N_{t}$. In comparison, the Alamouti's spacetime codes achieve a unit symbol rate, whereas the rate of higher-order orthogonal space-time block codes is lower than one.

Consider a simple situation where two transmit antennas are present, the following twolayer code provides symbol rate of two (i.e., full rate $R=2$ ),

$$
\mathbf{V}=\left[\begin{array}{ll}
\rho_{1} v_{1}^{(1)} & \rho_{2} v_{1}^{(2)} \\
\rho_{2} v_{2}^{(2)} & \rho_{1} v_{2}^{(1)}
\end{array}\right]
$$

The codeword matrix in (7) is mapped to the following general form of $2 \times 2$ space-time codeword,

$$
\mathbf{X}_{i}=\left[\begin{array}{ll}
x_{i}(1) & x_{i}(2) \\
x_{i}(3) & x_{i}(4)
\end{array}\right]=\sqrt{g} \mathbf{V}=\sqrt{g}\left[\begin{array}{ll}
\rho_{1} v_{1}^{(1)} & \rho_{2} v_{1}^{(2)} \\
\rho_{2} v_{2}^{(2)} & \rho_{1} v_{2}^{(1)}
\end{array}\right]
$$

where $g$ is a factor to normalize the averaging codeword energy. The multi-layer cyclotomic space-time codes are full-rate, and are shown in [20] to be of full diversity gain and optimal diversity product. When such space-time codes are used instead of the original data stream in an independent MIMO fading channel environment, therefore, improvements in system performance and power efficiency can be expected by taking the advantages of the full-rate and full-diversity features. 
The $2 \times 2$ full-rate cyclotomic space-time codes, described in (8), have the following important properties [20]

(P1) $\min \left|v_{1}^{(i)} v_{2}^{(i)}\right|=1, i=1,2$,

(P2) $\min \left|\operatorname{det}\left(\mathbf{V} \mathbf{V}^{H}\right)\right|=1$.

\subsection{High-Rate Full-Diversity Cooperation Schemes}

Figure 3 shows the proposed protocol. There are $M$ users present, each equipped with a single antenna that can be used for transmit and receive. When considering user $i$ as the source user, the other $M-1$ ones act as the relay users for user $i$. At the source user, a set of $M \times M$ multi-layer cyclotomic space-time codewords, $\mathbf{X}_{i}$, are formed from the source information sequence. As we discussed in Section 4.2, the original MIMO space-time codeword, before being modified for cooperative diversity, has a full rate (i.e., $R=M$ ). However, in the cooperative diversity system, the source user must first broadcast its information to the $M-1$ relay terminals because they do not have the information to be relayed. As such, the data rate in the cooperative diversity system is lower than that of the co-located MIMO counterpart.

In this protocol, the broadcast phase occupies $M-1$ time blocks, whereas the relay phase occupies one time block. In the broadcast phase, the source user transmits the $(l+1)$ th row of the $M \times M$ codeword through the $l$ th time block, where $l=1, \ldots, M-1$. In the relay phase occupying the $M$ th time block, the full codeword is transmitted from the $M$ users such that the $M-1$ relay terminals repeat the second through the $M$ th row of the codeword, whereas the first row of the codeword is transmitted from the source terminal.

For a $2 \times 2$ MIMO space-time codeword $\mathbf{X}_{i}$, the proposed protocol equivalently transmits the following $2 \times 4$ codeword $\mathbf{X}_{i}^{\prime}$, i.e.,

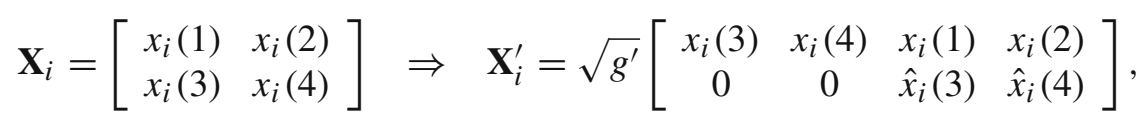

where $\hat{x}_{i}(t)$ is a complex scaled and noisy version of $x_{i}(t)$. The scale comes from the channel attenuation and the amplification in the relay terminal, whereas the relay noise is added before the amplification. In this protocol, the second row of the codeword $\mathbf{X}_{i}$ is first transmitted by the source terminal during the first time block and is then relayed by the relay terminal at the

\begin{tabular}{|c|c|c|c|c|c|}
\hline \multirow[b]{2}{*}{ Ch. 1} & \multicolumn{5}{|l|}{ A Channel allocations } \\
\hline & $\begin{array}{l}\text { user } 1 \text { transmits } 2 \text { nd } \\
\text { row of codeword } X_{1}\end{array}$ & $\begin{array}{l}\text { user } 1 \text { transmits } 3 \text { rd } \\
\text { row of codeword } X_{1}\end{array}$ & $\ldots \ldots$ & $\begin{array}{l}\text { user } 1 \text { transmits } \mathrm{M} \text {-th } \\
\text { row of codeword } \mathrm{X}_{1}\end{array}$ & $\begin{array}{l}\text { users } 1,2, \ldots, M \text { transmit } \\
\text { the entire codeword } X_{1}\end{array}$ \\
\hline \multirow[t]{2}{*}{ Ch. 2} & $\begin{array}{l}\text { user } 2 \text { transmits } 2 \text { nd } \\
\text { row of codeword } X_{2}\end{array}$ & $\begin{array}{l}\text { user } 2 \text { transmits } 3 \text { rd } \\
\text { row of codeword } X_{2}\end{array}$ & $\ldots \ldots$ & $\begin{array}{l}\text { user } 2 \text { transmits } \mathrm{M} \text {-th } \\
\text { row of codeword } \mathrm{X}_{2}\end{array}$ & $\begin{array}{l}\text { users } 1,2, \ldots, \mathrm{M} \text { transmit } \\
\text { the entire codeword } \mathrm{X}_{2}\end{array}$ \\
\hline & $\vdots$ & $\vdots$ & $\because$ & $\vdots$ & $\vdots$ \\
\hline Ch. M & $\begin{array}{l}\text { user } M \text { transmits 2nd } \\
\text { row of codeword } X_{M}\end{array}$ & $\begin{array}{l}\text { user } M \text { transmits 3rd } \\
\text { row of codeword } X_{M}\end{array}$ & $\cdots \cdots$ & $\begin{array}{l}\text { user } M \text { transmits } M-\text { th } \\
\text { row of codeword } X_{M}\end{array}$ & $\begin{array}{l}\text { users } 1,2, \ldots, M \text { transmit } \\
\text { the entire codeword } X_{M}\end{array}$ \\
\hline
\end{tabular}

Figure 3. The proposed space-time cooperation scheme. 
second time block. The first row of the codeword is transmitted from the source terminal at the second time block.

When we assume that $\hat{x}_{i}(t)$ is the exact replica of $x_{i}(t)$, we can equivalently consider the resulting codeword as

$$
\mathbf{X}_{i}^{\prime}=\sqrt{g^{\prime}}\left[\begin{array}{cccc}
x_{i}(3) & x_{i}(4) & x_{i}(1) & x_{i}(2) \\
0 & 0 & x_{i}(3) & x_{i}(4)
\end{array}\right]
$$

A code like $\mathbf{X}_{i}^{\prime}$ depicted in (12) is referred to as the distributed space-time code corresponding to the space-time code $\mathbf{X}_{i}$.

Compared to the existing space-time cooperative diversity schemes as depicted in Figure 2(b) and (c), the major difference of the proposed method is that the space-time encoding is performed at the source terminal instead of the relay terminals. As such, the proposed method permits space-time cooperative diversity using the relatively simple amplify-and-forward algorithm.

The expense of using the proposed protocol lies in the long time period it requires during the broadcast phase. This is true particularly when $M$ is large. The impact of this problem, however, can be reduced by using high-rate space-time codes. In the proposed method, a frame consisting of both broadcast and relay phases occupies $M^{2}$ symbol periods to deliver the distributed codeword $\mathbf{X}_{i}^{\prime}$. Note that codeword $\mathbf{X}_{i}^{\prime}$ contains all the $M^{2}$ elements of the $M \times M$ MIMO codeword $\mathbf{X}_{i}$, which, as can be clearly seen from equations (3), (6), (8) and (12), corresponds to $M^{2}$ information symbols. Therefore, when a full-rate space-time code is generated at the source terminal and is mapped to a distributed space-time code through the proposed protocol, the proposed method achieves unit DOF usage, which is compared to $1 / M$ (repetition-based diversity scheme in Figure 2(a)) and 1/2 (decode-based space-time cooperation schemes I and II in Figure 2(b) and (c)) in the aforementioned space-time cooperation schemes.

\section{Power Optimization}

The cyclotomic space-time codes were originally developed and optimized for transmit diversity in a co-located MIMO system, where the wireless channels between different transmit and receive antennas typically have the same statistics. In the underlying cooperative systems, however, the channels from different transmit antennas (i.e., different terminals) to the destination can be drastically different. In addition, different codeword elements are transmitted for different times during the broadcast and the relay phases. As a result, the distributed space-time code generated from an optimal space-time prototype may not be necessarily optimal. In this section, we consider a simple two-user scenario to show the importance of proper power optimization in the underlying cooperative networks.

Consider a $2 \times 2$ two-layer MIMO space-time code defined in (8). For simplicity, we assume that the channel between the source user and the relay user is dominated by time-invariant component (e.g., the source terminal and the relay terminal have a clear line-of-sight and the reflection and scattering is relatively low) and the relay noise is negligible. In this case, as we 
discussed in Section 4.3, the proposed protocol converts the codeword to an equivalent $2 \times 4$ distributed space-time codeword,

$$
\begin{aligned}
\mathbf{X}_{i}^{\prime} & =\sqrt{g^{\prime}}\left[\begin{array}{cccc}
x_{i}(3) & x_{i}(4) & \alpha x_{i}(1) & \alpha x_{i}(2) \\
0 & 0 & x_{i}(3) & x_{i}(4)
\end{array}\right] \\
& =\sqrt{g g^{\prime}}\left[\begin{array}{cccc}
\rho_{2} v_{2}^{(2)} & \rho_{1} v_{2}^{(1)} & \alpha \rho_{1} v_{1}^{(1)} & \alpha \rho_{2} v_{1}^{(2)} \\
0 & 0 & \rho_{2} v_{2}^{(2)} & \rho_{1} v_{2}^{(1)}
\end{array}\right]
\end{aligned}
$$

where a non-negative number $\alpha$ is introduced so that the power of transmitting $x_{i}(1)$ and $x_{i}(2)$ can be optimized. To maintain a unit codeword energy, we have $g g^{\prime}=\left[\left(2+\alpha^{2}\right)\left(\rho_{1}^{2}+\rho_{2}^{2}\right) \sigma^{2}\right]^{-1}$, where $\sigma^{2}$ is the average power of $v_{i}^{(k)}, i, k=1,2$, over all code constellations. Therefore, the determinant of the distributed codeword can be obtained as

$$
\begin{aligned}
\operatorname{det}\left[\mathbf{X}_{i}^{\prime}\left(\mathbf{X}_{i}^{\prime}\right)^{H}\right]= & \frac{1}{\left[\left(2+\alpha^{2}\right)\left(\rho_{1}^{2}+\rho_{2}^{2}\right) \sigma^{2}\right]^{2}} \\
& \times\left[\left(\left|\rho_{2} v_{2}^{(2)}\right|^{2}+\left|\rho_{1} v_{2}^{(1)}\right|^{2}\right)^{2}+\alpha^{2}\left|\rho_{1}^{2} v_{1}^{(1)} v_{2}^{(1)}-\rho_{2}^{2} v_{1}^{(2)} v_{2}^{(2)}\right|^{2}\right] .
\end{aligned}
$$

It is clear from (7) that $\rho_{1}^{2} v_{1}^{(1)} v_{2}^{(1)}-\rho_{2}^{2} v_{1}^{(2)} v_{2}^{(2)}$ in the above equation is the determinant of $\mathbf{V}$. Denote $\beta=\min \left(\left|\rho_{2} v_{2}^{(2)}\right|^{2}+\left|\rho_{1} v_{2}^{(1)}\right|^{2}\right)$ which is determined by the codeword. Then, from property $\mathrm{P} 2$ depicted in (10), the minimum of the above determinant becomes

$$
\min \operatorname{det}\left[\mathbf{X}_{i}^{\prime}\left(\mathbf{X}_{i}^{\prime}\right)^{H}\right]=\frac{\beta^{2}+\alpha^{2}}{\left[\left(2+\alpha^{2}\right)\left(\rho_{1}^{2}+\rho_{2}^{2}\right) \sigma^{2}\right]^{2}} .
$$

To maximize the above minimum determinant, therefore, the optimum value of $\alpha$ becomes

$$
\alpha_{\mathrm{opt}}=\sqrt{2\left(1-\beta^{2}\right)} .
$$

When the size of modulation constellations increases, $\beta$ decreases, and $\alpha$ asymptotically approaches $\sqrt{2}$.

\section{Numerical Results}

We consider a set of $2 \times 2$ cyclotomic space-time codes as the prototype for the development of the distributed space-time codes. The prototype codeword has 256 constellations to carry 8 bits of information over two time slots. This corresponds to QPSK modulated symbols of $u_{l}$ in equation (3). While we assume that there is no distortion between the source user and the relay terminal, the channels from both users to the destination are assumed to be independent and identically distributed (i.i.d.) random Gaussian processes with zero mean and unit variance. In addition, the relay noise is not considered in the simulations.

Figure 4 illustrates the minimum determinant of the codeword matrix versus the value of $\alpha$. The codewords are normalized such that the average energy of each distributed space-time 


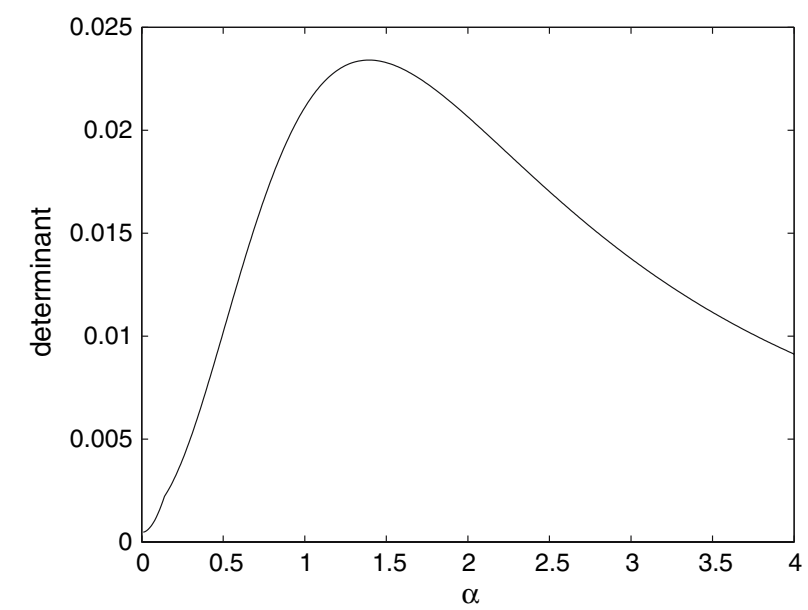

Figure 4. Minimum determinant of the distributed space-time codeword versus $\alpha$.

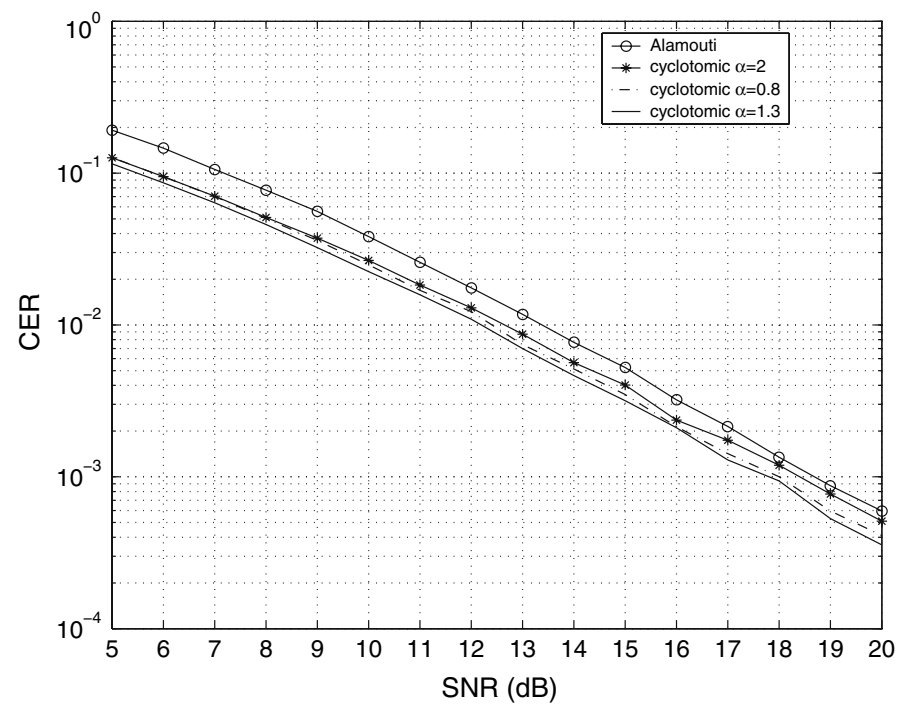

Figure 5. CER performance of the proposed scheme with different values of $\alpha$, compared with that developed from Almouti's space-time code.

codeword is one. It is evident from this result that the minimum determinant achieves its maximum value around $\alpha=\sqrt{2}$, and there is a $10 \%$ improvement in the minimum determinant at $\alpha=\sqrt{2}$ compared to that corresponding to $\alpha=1$.

Figure 5 compares the codeword error rate (CER) performance of the proposed scheme for $\alpha=0.8,1.3$, and 2. The SNR is defined as the ratio of the average codeword energy to the noise energy over the same period. For comparison, the results using the distributed spacetime codes based on Alamouti's space-time codes via the relationship of equation (12) are also computed. For fair comparison, each symbol in the Alamouti's code is modulated with 16 QAM so that the same 8 bits of information are carried over a two time-slot codeword 
period. It is seen that the result corresponding to $\alpha=1.3$ outperforms the others. The proposed method outperforms the cooperative code developed from Alamouti's scheme by about $1.5 \mathrm{~dB}$.

\section{Conclusions}

We have proposed a space-time cooperation scheme that exploits full-rate full-diversity cyclotomic space-time codes in the cooperative diversity platforms. The proposed protocol is applicable to both amplify-and-forward and decode-and-forward algorithms. The importance of power optimization within a distributed codeword is also discussed. The advantages of the proposed method were confirmed through numerical simulations.

\section{Acknowledgement}

This work was supported in part by the Office of Naval Research under Grant N00014-04-10617 and Contract No. N65540-05-C-0028.

\section{References}

1. G.J. Foschini and M.J. Gans, "On Limits of Wireless Communications in a Fading Environment when Using Multiple Antennas”, Wireless Personal Commun., Vol. 6, no. 3, pp. 311-335, March 1998.

2. V. Tarokh, H. Jafarkhani, and A.R. Calderbank, "Space-time Block Codes from Orthogonal Designs", IEEE Trans. Inform. Theory, Vol. 45, No. 5, July 1999.

3. S.M. Alamouti, "A Simple Transmitter Diversity Scheme for Wireless Communications", IEEE J. Select. Areas Commun., Vol. 16, pp. 1451-1458, Oct. 1998.

4. A.F. Naguib, N. Seshadri, and A.R. Calderbank, "Increasing Data Rate Over Wireless Channels: Space-Time Coding and Signal Processing for High Data Rate Wireless Communications", IEEE Signal Proc. Mag., Vol. 17, no. 3, pp. 76-92, May 2000.

5. A. Sendonaris, E. Erkip, and B. Aazhang, "Increasing Uplink Capacity Via User Cooperation Diversity", Proc. IEEE Int. Symp. Inform. Theory, Cambridge, MA, p. 156, Aug. 1998.

6. A. Sendonaris, E. Erkip, and B. Aazhang, "User Cooperative Diversity - Part I and Part II", IEEE Trans. Commun., Vol. 51, no. 11, pp. 1927-1948, Nov. 2003.

7. J.N. Laneman, D.N.C. Tse, and G.W. Wornell, "Cooperative Diversity in Wireless Networks: Effective Protocols and Outrage Behavior”, IEEE Trans. Inform. Theory, Vol. 50, no. 12, pp. 3062-3080, Dec. 2004.

8. J.N. Laneman and G.W. Wornell, "Distributed Space-Time Coded Protocols for Exploiting Cooperative Diversity in Wireless Networks", IEEE Trans. Inform. Theory, Vol. 49, No. 10, pp. 2415-2425, Oct. 2003.

9. A.F. Dana and B. Hassibi, "On the Power Effeciency of Sensory and Ad-Hoc Wireless Networks", IEEE Trans. Inform. Theory, Vol. 52, No. 7, pp. 2890-2914, July 2006.

10. P.A. Anghel, G. Leus, and M. Kaveh, "Multi-User Space-Time Coding in a Cooperative Networks", Proc. IEEE ICASSP, Hong Kong, May 2003.

11. Y. Hua, Y. Mei, and Y. Chang, "Parallel Wireless Mobile Relays with Space-Time Modulations", Proc. IEEE Workshop on Statistical Signal Processing, St. Louis, MO, Sept. 2003.

12. G. Wang, Y. Zhang, and M.G. Amin, "Differential Distributed Space-Time Modulation for Cooperative Networks", IEEE Trans. Wireless Commun., in press.

13. M. Janani, A. Hedayat, T.E. Hunter, and A. Nosratinia, "Coded Cooperative in Wireless Communications: Space-Time Transmission and Iterative Decoding”, IEEE Trans. Signal Proce., Vol. 52, no. 2, pp. 362-371, Feb. 2004. 
14. M.O. Damen, A. Tewfik, and J.-C. Belfiore, "A construction of a Space-Time Code Based on the Theory of Numbers", IEEE Trans. Inform. Theory, Vol. 48, No. 3, pp. 753-760, March 2002.

15. H. El Gamal and M.O. Damen, "Universal Space-Time Coding", IEEE Trans. Inform. Theory, Vol. 49, pp. 1097-1119, May 2003.

16. M.O. Damen, and N.C. Beaulieu, "On Two High Rate Algebraic Space-Time Codes”, IEEE Trans. Inform. Theory, Vol. 49, pp. 1059-1063, April 2003.

17. M.O. Damen, H. El Gamel, and N.C. Beaulieu, "Linear Threaded Algebraic Space-Time Constellations", IEEE Trans. Inform. Theory, Vol. 49, No. 10, pp. 2372-2388, Oct. 2003.

18. X. Ma and G.B. Giannakis, "Full-Diversity Full Rate Complex-Field Space-Time Coding”, IEEE Trans. Signal Proce., Vol. 51, No. 11, Nov. 2003.

19. G. Wang, H. Liao, H. Wang, and X.-G. Xia, "Systematic and Optimal Cyclotomic Space-Time Code Designs Based on High Dimensional Lattices", Proc. Globecom, San Fransicso, CA, Dec. 2003.

20. G. Wang and X.-G. Xia, "On Optimal Multilayer Cyclotomic Space-Time Code Designs", IEEE Trans. Inform. Theory, Vol. 51, No. 3, pp. 1102-1135, March 2005.

21. J.-C. Belfiore, G. Rekaya, and E. Viterbo, "The Golden Code: A $2 \times 2$ Full-Rate Space-Time Code with Non-Vanishing Determinants", Proc. IEEE Int. Symp. Inform. Theory, Chicago, IL, June/July 2004.

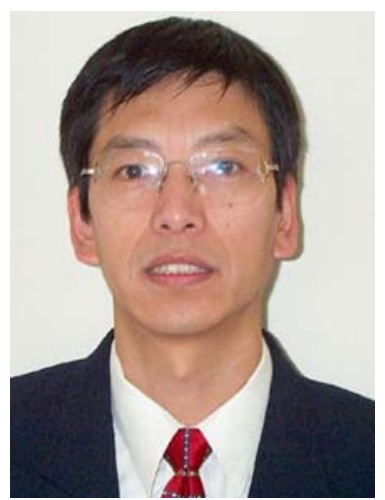

Genyuan Wang received the B.Sc. and M.S. degrees in mathematics from Shaanxi Normal University, Xi' an, China, in 1985 and 1988, respectively. He received his Ph.D. degree in electrical engineering from Xidian University, Xi' an China, in 1998.

From 1988 to 1994, he worked at Shaanxi Normal University as an assistant professor and then an associate professor. From 1994 to 1998, he worked at Xidian University as a research assistant. He worked with the Department of Electrical and Computer Engineering, University of Delaware as a post-doctoral fellow. Currently, he works with the Center of Advanced Communications, Villanova University, as a research associate. His research interests are radar imaging, radar signal processing, adaptive filter, OFDM system, channel equalization, channel coding, space-time coding, and multiple-input multiple-output for broadband wireless communication systems. 


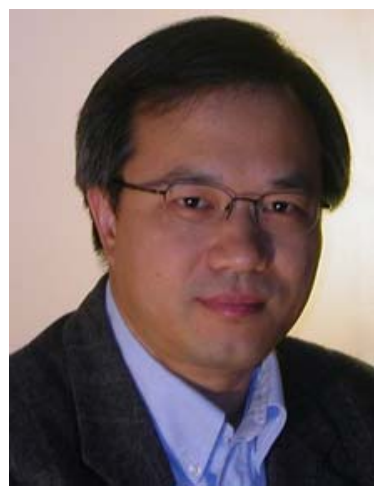

Yimin Zhang received his Ph.D. degrees from the University of Tsukuba, Tsukuba, Japan, in 1988. He joined the faculty of the Department of Radio Engineering, Southeast University, Nanjing, China, in 1988. He served as a Technical Manager at the Communication Laboratory Japan, Kawasaki, Japan, from 1995 to 1997, and was a Visiting Researcher at ATR Adaptive Communications Research Laboratories, Kyoto, Japan, from 1997 to 1998. Since 1998, he has been with the Villanova University, where he is currently a Research Associate Professor at the Center for Advanced Communications and the Director of the Radio Frequency Identification (RFID) Lab.

Dr. Zhang's research interests are in the areas of array signal processing, space-time adaptive processing, wireless networks, MIMO systems, cooperative diversity, blind signal processing, digital mobile communications, time-frequency analysis, source localization and tracking. He is an Associate Editor for IEEE signal processing Letters.

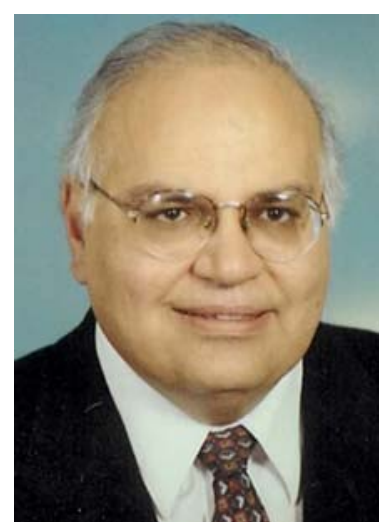

Moeness Amin received his Ph.D. degree in 1984 from University of Colorado, Boulder. He has been on the Faculty of Villanova University since 1985, where is now a Professor in the Department of Electrical and Computer Engineering and the Director of the Center for Advanced Communications.

Dr. Amin is the recipient of the IEEE Third Millennium Medal; Distinguished Lecturer of the IEEE Signal Processing Society for 2003-2004, Member of the Franklin Institute Committee on Science and the Arts; Recipient of the 1997 Villanova University Outstanding Faculty Research Award; Recipient of the 1997 IEEE Philadelphia Section Service Award. 
Dr. Amin has over 300 publications in the areas of Wireless Communications, Time-Frequency Analysis, Smart Antennas, Interference Cancellation in Broadband Communication Platforms, Digitized Battlefield, Direction Finding, Over the Horizon Radar, Radar Imaging, and Channel Equalizations. 